13

\title{
Влияние давления аргона и отжига на микрокристаллическую структуру текстурированных пленок Со, осаждаемых магнетронным распылением
}

\author{
(C) А.С. Джумалиев, ${ }^{1,2}$ Ю.В. Никулин, ${ }^{1,2}$ Ю.А. Филимонов ${ }^{1,2,3}$ \\ ${ }^{1}$ Саратовский филиал Института радиотехники и электроники им. В.А. Котельникова РАН, \\ 410019 Саратов, Россия \\ ${ }^{2}$ Саратовский национальный исследовательский государственный университет им. Н.Г. Чернышевского, \\ 410012 Саратов, Россия \\ ${ }^{3}$ Саратовский государственный технический университет им. Ю.А. Гагарина, \\ 410054 Саратов, Россия \\ e-mail: yvnikulin@gmail.com
}

(Поступило в Редакцию 5 декабря 2017 г.)

Исследовано влияние давления аргона $P(0.13 \leq P \leq 1 \mathrm{~Pa})$ и отжига в вакууме на микроструктуру и текстуру пленок кобальта толщиной $d \approx 300 \mathrm{~nm}$, полученных методом магнетронного распыления на подложках $\mathrm{SiO}_{2} / \mathrm{Si}$. Показано, что пленки, осаждаемые при давлении $0.26 \leq P<1 \mathrm{~Pa}$, характеризуются столбчатой микроструктурой по толщине и смешанной кристаллической фазой hcp-Co(002)/fcc-Co $(111)$. Отжиг приводит к более однородному микроструктурному строению за счет увеличения размеров зерен и улучшает текстуру hср-Co(002)/fcc-Co(111). Пленки, осаждаемые при $0.13 \leq P<0.18$ Ра, имеют смешанную кристаллическую фазу - помимо фаз hcp-Co(002)/fcc-Co(111) и hcp-Co(101) присутствует кристаллическая фаза с гранецентрированной кубической кристаллической решеткой и текстурой fcc-Co(200). Пленки, выращенные при $P \approx 0.13 \mathrm{~Pa}$, характеризуются текстурой fcc-Co $(200)$ и неоднородным микроструктурным строением по толщине - на границе с подложкой, в слое толщиной $d_{c} \approx 100-130 \mathrm{~nm}$, пленки имеют квазиоднородную микроструктуру, которая на толщинах $d>d_{c}$ переходит в „гранулированную“. Отжиг таких пленок приводит к более однородному микроструктурному строению за счет увеличения размеров зерен, улучшает текстуру fcc-Co(200) и приводит к появлению кристаллической фазы fcc-Co(111)/hcp-Co(002).

DOI: 10.21883/JTF.2018.11.46638.2586

\section{Введение}

Механизмы формирования кристаллической текстуры и микроструктурного строения пленок кобальта (Со) активно изучаются как с практической, так и с фундаментальной точек зрения. Этот интерес связан прежде всего с перспективами широкого применения пленок и многослойных структур Со и сплавов на его основе при разработке сред для перпендикулярной магнитной записи $[1,2]$, решеток наночастиц для сверхплотной записи [3], а также магнитной памяти с произвольным доступом [1]. Туннельные [4] и многослойные структуры [5] на основе пленок Со демонстрируют высокое магнитосопротивление, что делает пленки Со одним из основных материалов спинтроники [6]. Кроме того, пленки кобальта широко используются в качестве катализаторов при осаждении углеродных нанотрубок [7] и графена [8], в производстве биотоплива [9].

С фундаментальной точки зрения, пленки кобальта представляют интерес в качестве удобной модели для изучения структурно-зависимых магнитных эффектов, a также явлений, чувствительных к энергетическим характеристикам поверхности. Пленки Со демонстрируют метастабильные гранецентрированные (fcc) [10-12] и объемноцентрированные (bcc) $[10,12,13]$ кубические кристаллические фазы и стабильные гексагональные плотноупакованные (hcp) фазы в зависимости от условий роста и подложки. Текстура пленок кобальта оказывает влияние на протекание каталитических $[14,15]$ и твердофазных [16] химических реакций. Использование в качестве спин-поляризационного электрода пленок bcc-Co(001) позволяет поднять туннельное магнитосопротивление структур $\mathrm{Co} / \mathrm{MgO} / \mathrm{Co}$ до 400\% [17]. Кроме того, текстура и микроструктурное строение пленок кобальта оказывают существенное влияние на величину поля перпендикулярной магнитной анизотропии слоистых структур $\mathrm{Co} / \mathrm{Pd}[18]$ и $\mathrm{Co} / \mathrm{Ni}$ [19], а также на величину и однородность поля перемагничивания решеток магнитных наночастиц $\mathrm{Co} / \mathrm{Pd}[20]$.

Пленки кобальта могут быть получены с помощью различных технологий осаждения: молекулярно-лучевой эпитаксии [11], ионно-лучевым распылением [21], электронно-лучевым испарением [22,23], магнетронным распылением [24-26], электролитическим осаждением $[27,28]$ и химическим осаждением из газовой фазы металлоорганических соединений [29]. При этом на формирование текстуры и микроструктурное строение пленок оказывают влияние, прежде всего, параметры подложки [30,31] и осаждения [21-29] пленок, а также толщина $[13,32]$ и послеростовой отжиг $[21,23,33]$ пленки. 
Применительно к пленкам Со, полученным методом магнетронного распыления на неориентирующих подложках [34], исследование механизмов формирования и модификации текстуры и микроструктурного строения проводилось в работах $[24,25,30,33,35-39]$. При этом обсуждались влияния температуры подложки $T_{s}$ [35], температуры отжига $T_{a}[33,36]$, типа подслоя [30] и бомбардировки пленки потоком ионов [38]. Было показано, что пленки Со формируются, как правило, с двумя кристаллическими фазами: $\mathrm{hcp}-\mathrm{Co}(002)$ и $\mathrm{fcc}-\mathrm{Co}(111) \quad[35,36,24,38]$. Однако при определенных условиях наблюдалось формирование пленок с кристаллической фазой fcc-Co(200) [35,24,38]. Например, в $[35,24]$ отмечалось, что в пленках толщиной $d \approx 123-300 \mathrm{~nm}$, выращенных радиочастотным распылением на „горячих“ подложках с температурой $T_{s}$ выше комнатной, обнаруживается присутствие кристаллитов $\mathrm{fcc-Co}(200)$. При этом в работе [24] было показано, что в пленках толщиной $d \approx 12-25 \mathrm{~nm}$ кристаллическая фаза $\mathrm{fcc}-\mathrm{Co}(200)$ может стать доминирующей в узком диапазоне температур $( \pm 50 \mathrm{~K})$ вблизи $T_{s} \approx 550 \mathrm{~K}$. Также было показано [38], что воздействие потока ионов золота с энергией $320 \mathrm{keV}$ на поликристаллическую пленку hср-Со приводит к структурному изменению из hcp-Со в текстурированную fcc-Co(200). Вместе с тем на сегодняшний день возможность формирования текстурированных пленок кобальта fcc-Co(200) на неориентирующих подложках при комнатной температуре методом магнетронного распыления на постоянном токе не обсуждалась.

В настоящей работе показана возможность формирования на подложках $\mathrm{SiO}_{2} / \mathrm{Si}$, находящихся при комнатной температуре, текстурированных пленок кобальта толщиной $d \approx 300 \mathrm{~nm}$ с термически стабильной доминирующей кристаллической фазой $\mathrm{fcc}-\mathrm{Co}(200)$ за счет снижения давления рабочего газа до значений $P \approx 0.13 \mathrm{~Pa}$, при которых режим пролета распыленных атомов Со становится бесстолкновительным и обеспечивает высокую миграционную способность адатомов по подложке [40]. Также исследовано влияние послеростового отжига в вакууме при $T_{a} \approx 650 \mathrm{~K}$ на кристаллическое и микроструктурное строение полученных пленок $\mathrm{Co} / \mathrm{SiO}_{2} / \mathrm{Si}$.

\section{1. Результаты и обсуждение}

Осаждение пленок кобальта производилось в магнетронной распылительной системе на постоянном токе с базовым давлением $0.2 \mathrm{mPa}$. Для напыления использовались мишени из кобальта чистотой $99.95 \%$ (Williams) и аргон марки ОЧ (99.998\%). Подложка размещалась по центру мишени на расстоянии $L \approx 100 \mathrm{~mm}$. Напряжение на мишени составляло $U \approx-500 \mathrm{~V}$. В качестве подложек использовались пластины $\mathrm{Si}(100)$ с термически окисленным слоем $\mathrm{SiO}_{2}$ толщиной $300 \mathrm{~nm}$ и среднеквадратичной амплитудой шероховатости поверхности $\sigma \approx 0.3 \mathrm{~nm}$. Подложки подвергались ультразвуковой очистке в ацетоне и непосредственно перед напылением термическому отжигу в вакууме при $T_{a} \approx 650$ в течение $30 \mathrm{~min}$. Пленки осаждались на „заземленную“ подложку (напряжение смещения на подложке $U_{s} \approx 0$ ) при температуре $T_{s} \approx 300 \mathrm{~K}$ и давлении аргона $P \approx 0.13,0.18$, $0.26,0.4,0.67$ и $1 \mathrm{~Pa}$, а также при $T_{s} \approx 77 \mathrm{~K}$ и $P \approx 0.13 \mathrm{~Pa}$. Скорость напыления составляла $v \approx 9-14 \mathrm{~nm} / \mathrm{min}$ для давления аргона $P \approx 1-0.13$ Ра. После напыления часть пленок подвергалась отжигу в вакууме в течении 30 min при температуре $T_{a} \approx 650 \mathrm{~K}$, ниже температуры мартенситного перехода $\mathrm{hcp}(002)-\mathrm{fcc}(111)$ для кобальта $\left(T_{m} \approx 700 \mathrm{~K}\right)$. Отжиг при выбранной $T_{a}$ позволял добиваться изменения кристаллической структуры пленок в отсутствии вклада от мартенситного перехода.

Кристаллическая структура пленок изучалась методом рентгеновской дифракции на дифрактометре ДРОН-4 с фокусировкой по плоскому образцу в геометрии Брэгга-Брентано (схема $\Theta-2 \Theta \mathrm{Cu}-K \alpha$ излучение, $\lambda \approx 0.15418 \mathrm{~nm}$ ). Величина межплоскостного расстояния $d_{[\mathrm{hkl}]}$ вдоль кристаллографической оси $[\mathrm{hkl}]$, совпадающей с направлением нормали к пленке, определялась по формуле Брэгга-Вульфа $2 \cdot d_{[\mathrm{hkl}]} \cdot \sin \Theta \approx \lambda$, где $\Theta-$ угол падения излучения, отсчитываемый от плоскости пленки. Из сравнения положения экспериментальных дифракционных линий и линий эталонного порошка Со (база данных Международного Центра по дифракционным данным, карточки № 15-0806, № 05-0727) было установлено, что в зависимости от ростовых условий в пленках формируются кристаллические фазы с гексагональной плотноупакованной (hcp) и гранецентрированной кубической (fcc) кристаллической структурой. Морфология поверхности пленок исследовалась методами атомно-силовой микроскопии (АСM), среднеквадратичная шероховатость поверхности $\sigma$ пленок определялась с помощью программного обеспечения микроскопа Solver P-47 (NT-MDT). Микроструктура пленок изучалась методом сканирующей электронной микроскопии (СЭМ) (Auriga, Carl Zeiss). Толщина пленок определялась методом профилометрии (Dectak 150, Veесо) с точностью 5\%. Приведенные в настоящей работе результаты получены для пленок толщиной $d \approx 300 \mathrm{~nm}$. Такой выбор толщины обусловлен высокой чувствительностью микроструктурного строения и текстуры поликристаллических пленок к упругим напряжениям, которые вызваны дефектами упаковки, что, в частности, проявляется для пленок $\mathrm{Co} / \mathrm{SiO}_{2} / \mathrm{Si}(100)$ при фазовом переходе hcp-fcc [22]. C ростом толщины пленки упругие напряжения накапливаются $[41,42]$ и в ряде случаев могут достигать насыщения при некоторой толщине пленки $d \approx d_{c}$ [42]. Ранее для пленок $\mathrm{Ni} / \mathrm{SiO}_{2} / \mathrm{Si}(100)$, полученных при таких же, как в настоящей работе, технологических параметpax магнетронного распыления, мы наблюдали перестройку микроструктурного строения по толщине в с $d>d_{c} \approx 130-150 \mathrm{~nm}[40,43]$. 


\section{1. Влияние давления аргона на микрокристаллическую структуру пленок}

На рис. 1 приведены дифрактограммы пленок кобальта толщиной $d \approx 300 \mathrm{~nm}$, осажденных при различных давлениях рабочего газа $P$. Из рисунка видно, что осажденные при $P \approx 0.26-1$ Ра пленки имеют смешанную кристаллическую фазу hcp-Co(002)/fcc-Co(111), которая характеризуется минимальной поверхностной энергией $\gamma\left(\gamma_{\mathrm{hcp}}^{(002)} \approx 3.18 \mathrm{~J} / \mathrm{m}^{2}, \gamma_{\mathrm{fcc}}^{(111)} \approx 3.23 \mathrm{~J} / \mathrm{m}^{2}[44,45]\right)$. Интенсивность дифракционного пика hcp-Co(002)/fcc-Co(111) слабая, что согласуется с результатами работы [30]. Отметим, что поверхностная энергия подложки $\mathrm{SiO}_{2}$ составляет $\gamma^{\mathrm{SiO}_{2}} \approx 0.253 \mathrm{~J} / \mathrm{m}^{2} \quad[46]$, и в нашем случае выполняется условие $\left(\gamma^{\mathrm{SiO}_{2}} \ll \gamma^{\mathrm{Co}}\right)$ трехмерной модели роста пленок Со $[47,30]$. То обстоятельство, что, помимо hсp-Co(002) кристаллической фазы с наименьшим значением $\gamma$, присутствуют зерна fcc-Co(111), объясняется близостью значений поверхностных энергий $\left(\gamma_{\mathrm{hcp}}^{(002)} \approx \gamma_{\mathrm{fcc}}^{(111)}\right)$ кристаллитов hcp-Co(002) и fcc-Co(111). Кроме того, на границах кристаллитов hcp-Co(002)/fcc-Co(111) происходит уменьшение координационного числа атомов $\mathrm{Co}$, что может снижать энергию дефектов упаковки на границах зерен (ГЗ) относительно границ зерен одинаковой кристаллической фазы (hcp-hcp или fcc-fcc) и способствовать минимизации плотности энергии пленки [48].

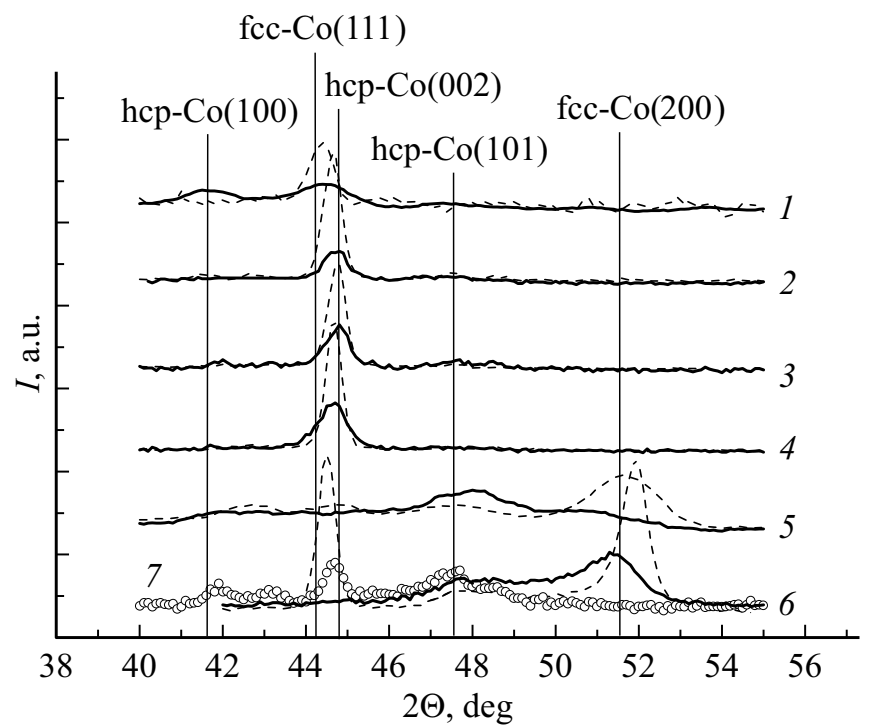

Рис. 1. Дифрактограммы пленок Со толщиной $d \approx 300 \mathrm{~nm}$, полученных при различном давлении рабочего газа при температуре подложки $T_{s} \approx 300 \mathrm{~K}$ до отжига (сплошные линии) и после отжига при $T_{a} \approx 650 \mathrm{~K}$ (штриховые линии) $P$, Ра: $1-$ $\approx 1,2-\approx 0.67,3-\approx 0.4,4-\approx 0.26,5-\approx 0.18$, $6-\approx 0.13$. Кривая 7 - дифрактограмма пленки, выращенной при $T_{s} \approx 77 \mathrm{~K}, P \approx 0.13$ Ра. Вертикальными линиями обозначены положения дифракционных линий эталонного порошка Со (база данных Международного Центра по дифракционным данным, карточки № 15-0806, № 05-0727).
Снижение давления рабочего газа до $P \approx 0.18 \mathrm{~Pa}$ приводит к формированию широкого дифракционного пика с максимумом вблизи положения линии от кристаллической фазы hcp-Co(101), в который также дают вклад дифракционные линии, отвечающие fcc-Co(200) и hср-Co(002). При $P \approx 0.13 \mathrm{~Pa}$ кристаллическая фаза fcc-Co(200) становится доминирующей, о чем говорит превышение интенсивности дифракционной линии fcc-Co(200) над интенсивностью линии hсp-Co(101) в 2.2 раза. При этом в спектре отсутствует дифракционная линия, отвечающая кристаллической фазе hcp-Co(002)/fcc-Co(111).

Изменение кристаллического строения и текстуры пленки с hcp-Co(002)/fcc-Co(111) на fcc-Co(200) при снижении $P$ обусловлено энергетическими процессами, происходящими с атомами кобальта как в пространстве между распыляемой мишенью и подложкой, так и на поверхности подложки. Действительно, длина свободного пробега атома Со в пространстве между мишенью и подложкой при $P \approx 1 \mathrm{~Pa}$ составляет около $5 \mathrm{~mm}$, тогда как при $P \approx 0.13 \mathrm{~Pa}-$ около $55 \mathrm{~mm}$. Поэтому при высоком давлении каждый из распыленных атомов Со испытывает около 20 столкновений в промежутке между мишенью и подложкой, что ведет к потерям энергии. Вследствие этого, миграционная способность (МС) адатомов на поверхности подложки снижается, что способствует образованию зародышей наименьшего размера [49]. При низком $P$ режим пролета атомов становится практически бесстолкновительным (происходит в среднем около 2 столкновений), что ведет не только к увеличению МС адатомов, но и к большему по сравнению с $P \approx 0.26-1$ Ра нагреву формирующейся пленки [34].

Стадии формирования кристаллических плоскостей fcc-Co(200) и hcp-Co(002) схематически показаны на рис. 2 (плоскость fcc-Co(111) формируется аналогично hср-Co(002) [50]). При низкой МС адатомов Со на подложке $(P \approx 1-0.26 \mathrm{~Pa})$ наиболее устойчивым из зародышей, образование и рост которых приводит к формированию кристаллитов hср-Co(002) (или fcc-Co(111)) (рис. 2, $a, c)$, является кластер А из трех атомов, на каждый атом в котором приходится по 2 одинаковые связи. В процессе роста кластер А может присоединить к себе еще один атом и стать кластером из четырех атомов, расположенных в виде ромба или пирамиды (кластеры В), увеличив тем самым свою энергию связи [51]. Дальнейшее увеличение размеров кластеров В приведет к дальнейшему увеличению энергии связи, увеличению стабильности кластеров [51] и в итоге к образованию кристаллической фазы hcp-Co(002)/fcc-Co(111).

При высокой $\mathrm{MC}$ адатомов на подложке $(P \approx$ $\approx 0.18-0.13 \mathrm{~Pa})$ одним из наиболее устойчивых кластеров малого размера, формирование и рост которых может привести к образованию кристаллитов fcc-Co(200), является кластер С (рис. 2,d). Такой кластер является менее устойчивым с точки зрения энергии связи $E_{b}$ по сравнению с кластером 

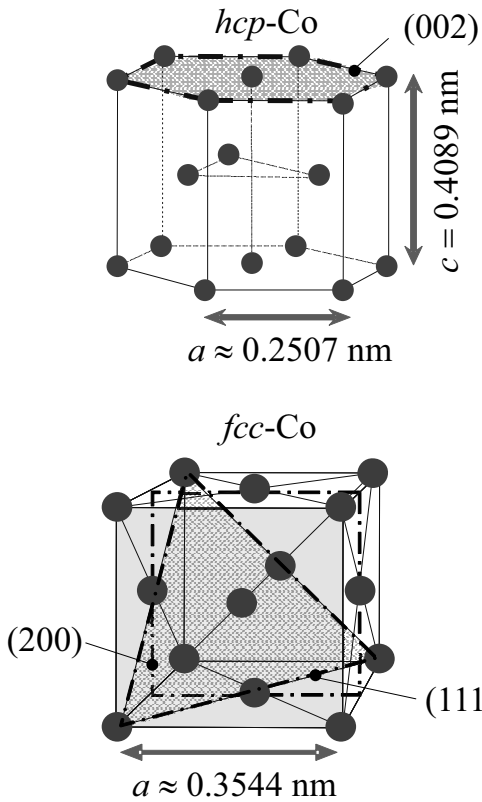

(002)

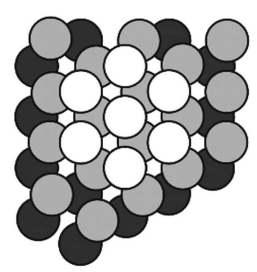

(111)

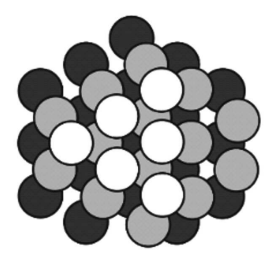

$a$

The 1-st layer of atoms

2-nd layer of atoms

The 3-rd layer of atoms $b$

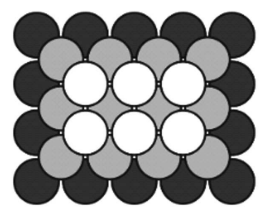

$E_{b} \approx 3.113$

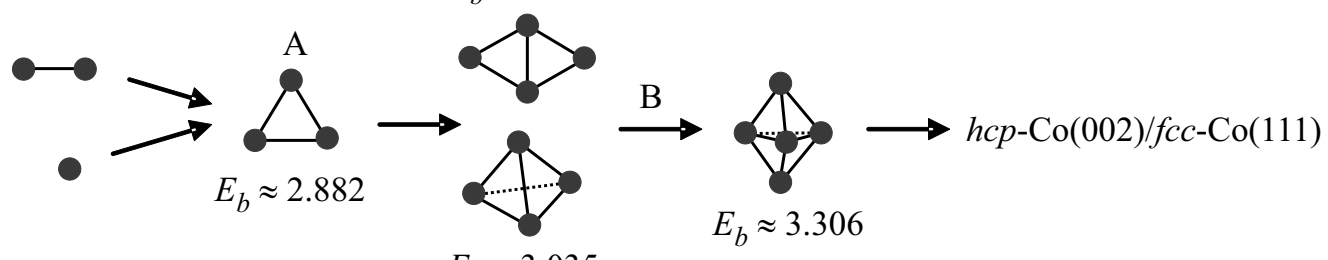

$E_{b} \approx 3.035$

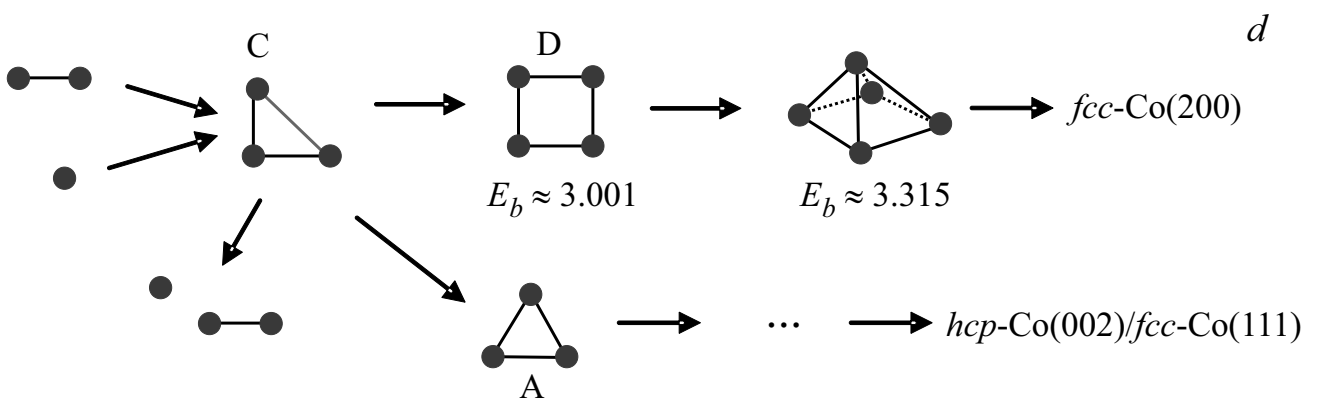

Рис. 2. $a, b-$ изображение гексагональной плотноупакованной (hcp) кристаллической решетки Со $(a)$ и гранецентрированной кубической $(\mathrm{fcc})$ решетки Со $(b)$ и кристаллографических плоскостей hcp-Co(002), fcc-Co(111) и fcc-Co(200); $c, d-$ этапы образования кластеров, рост которых приводит к формированию текстуры $(c)$ hcp-Co(002)/fcc-Co(111) и $(d)$ fcc-Сo(200). Рядом с изображениями кластеров указаны энергии связи $E_{b}(\mathrm{eV} / \mathrm{atom})[15]$.

А [51]. Он может распасться на димер и мономер, либо перестроиться в кластер А, либо до того, как распадется, может успеть присоединить к себе один атом Со и стать устойчивым кластером $\mathrm{D}$, дальнейший рост которого приведет к формированию кристаллитов fcc-Co(200). Таким образом, условием формирования кристаллитов fcc-Co(200) является высокая миграционная способность адатомов по подложке. При высоком давлении рабочего газа (низкая MC) кластеры D не успевают зарождаться, и в пленке формируется только смешанная кристаллическая фаза $\mathrm{hcp}-\mathrm{Co}(002) / \mathrm{fcc}-\mathrm{Co}(111)$ с наименьшей поверхностной энергией $[44,45]$.
В качестве подтверждения данного предположения на рис. 1 приведена дифрактограмма пленки, осажденной при $P \approx 0.13 \mathrm{~Pa}$ и $T_{s} \approx 77 \mathrm{~K}$. При этом ожидалось, что снижение температуры подложки до температуры жидкого азота ограничит МС адатомов, и образование кристаллической фазы fcc-Co(200), как и в случае высокого давления, будет затруднено. Как видно из рис. 1, в этом случае действительно формируются поликристаллические пленки с преобладанием текстуры hcp-Co(002) и $\mathrm{hcp}-\mathrm{Co}(101)$, тогда как кристаллическая фаза fcc-Co(200) не формируется.

Отметим, что формирование пленок fcc-Co $(200)$ с точки зрения поверхностной энергии $\gamma$ является ме- 
нее выгодным по сравнению с кристаллическими фазами hср-Co(002) и fcc-Co(111): $\gamma_{\mathrm{fcc}}^{(002)}>\gamma_{\mathrm{fcc}}^{(111)}>\gamma_{\mathrm{hcp}}^{(002)}$ $\left(\gamma_{\mathrm{fcc}}^{(200)} \approx 3.40 \mathrm{~J} / \mathrm{m}^{2}[44,45]\right)$. Однако в случае формирования кристаллической фазы fсc-Co(200) при $P \approx 0.13 \mathrm{~Pa}$ и $T_{s} \approx 300 \mathrm{~K}$, минимизация полной энергии пленки и соответственно стабилизация кристаллической фазы fcc-Co(200) может происходить за счет минимизации энергии упругих напряжений [52], возникающих в процессе роста пленки. Принимая во внимание, что для пленок fcc-Co(200) значение модуля Юнга $E \approx 100 \mathrm{GPa}$ меньше, чем для пленок hср-Сo $(E \approx 280-380 \mathrm{GPa})$ [33], следует ожидать, что кристаллиты fcc-Сo легче поддаются деформациям сжатия или растяжения и тем самым более эффективно минимизируют возникающие в процессе роста пленки упругие напряжения.

Кроме того, дополнительным фактором, способным компенсировать проигрыш в поверхностной энергии при формировании пленок с текстурой fcc-Co(200), может выступать снижение энергии дефекта упаковки на ГЗ $[48,53]$. Присутствие в дифрактограммах пленок, осажденных при низком давлении, рефлекса от кристаллитов hср-Co(101) (рис. 1) также следует связать с минимизацией энергии упругих деформаций һср-кристаллитов в пирамидальной плоскости (101), для которой модуль Юнга $(E \approx 280 \mathrm{GPa})$ имеет наименьшее значение среди других плоскостей hср-Со [33] .

Отметим, что наряду с изменением текстуры и кристаллического строения, снижение $P$ с 1 до $0.13 \mathrm{~Pa}$ приводит также к изменению микроструктурного строения пленок кобальта. Осаждение при высоком давлении рабочего газа $(1-0.26 \mathrm{~Pa})$ способствует преимущественному росту кристаллитов в направлении поступления атомов, что при низкой МС адатомов приводит к формированию столбчатой структуры пленки (рис. 3). Рассуждения о влиянии низкой МС на формирование столбчатой структуры подтверждаются изображением скола пленки, осажденной при $P \approx 0.13 \mathrm{~Pa} \mathrm{и} T_{s} \approx 77 \mathrm{~K}$ (низкая МС), представленного на вставке к рис. 3, $a 4$. Из рисунка видно, что в этом случае также происходит формирование столбчатой структуры.

Снижение давления рабочего газа до $P \approx 0.13$ Ра приводит к тому, что пленки формируются с „переходным“ типом микроструктурного строения - на начальных этапах роста образуется квазиоднородная микроструктура, которая изменяется на „гранулированную“ при достижении пленкой критической толщины $d_{c}$, величина которой определяется условиями осаждения, в частности температурой подложки. Нужно отметить, что „переходный толщине ранее наблюдался для текстурированных пленок $\mathrm{Ni}(200)$ с fcc кристаллической структурой [40,43], и его формирование может быть связано с конкурирующим воздействием на кинетику роста пленки процессов нестабильности скорости роста fсс и hср кристаллитов из-за эффекта самозатенения и ограничения поверхностной диффузии [54,55]. При этом к ограничению
MC адатомов могут приводить потенциальные барьеры, формирующиеся за счет упругой энергии дефектов упаковки на ГЗ. Неоднородность микроструктурного строения пленок, выращенных при „низком“ давлении (рис. 3, a 4), вызвана неоднородными упругими деформациями по толщине пленки. С упругими деформациями как в плоскости, так и в направлении нормали к пленке можно связать появление дифракционного максимума hcp-Co(101), который отражает сдвиг атомов кобальта в пирамидальной плоскости hср кристаллической решетки, направленной под углом $\approx 60^{\circ}$ к плоскости hср-Сo(001). На присутствие упругих деформаций кристаллитов также указывает смещение максимумов дифракционных линий fcc-Co(200) и hсp-Co(101) при $P \approx 0.13$ и $\approx 0.18$ Ра относительно эталонных и их значительная ширина (рис. 1). Последнее обстоятельство проявляется в расхождении оценок среднего размера зерна $D$, полученных, с одной стороны, из обработки изображений СЭМ и АСМ и, с другой, по формуле Шерpepa [56]:

$$
D \approx \frac{K \lambda}{\beta \cos \theta}
$$

где $K$ - константа Шерера, зависящая от формы кристаллитов и их распределения по размерам, порядка дифракционного максимума и способа определения ширины дифракционного пика, $\beta$ - ширина рефлекса на полувысоте (в радианах), $\theta-$ угол дифракции (брэгговский угол). Если считать форму кристаллитов близкой к сферической $(K \approx 1)$, то для случая $\mathrm{Cu}-K \alpha$ излучения $(\lambda \approx 0.15418 \mathrm{~nm})$ и рассматриваемых на рис. 1 углов $\theta$, формула (1) может быть представлена в виде:

$$
D(\mathrm{~nm}) \approx \frac{10 \mathrm{~nm}}{\Delta(2 \theta)}
$$

где $\Delta(2 \theta)$ - ширина пика по уровню $1 / 2$ в градуcax, которая для дифракционных линий fcc-Co(200) и hср-Сo(101) при $P \approx 0.13$ и $\approx 0.18$ Ра на рис. 1 составляет $\Delta(2 \theta) \approx(1.7-2)^{\circ}$. С учетом этого из формулы $(2)$ получаем оценку среднего размера гранул $D \approx 5-6 \mathrm{~nm}$, тогда как обработка СЭМ (см. рис. $3, c, d$ ) изображений дает значения $D \approx 25-50 \mathrm{~nm}$.

Изменение микроструктурного строения пленок Со со столбчатого на „переходный“ проявляется в снижении шероховатости поверхности $\sigma$ с 3.2 до $0.84 \mathrm{~nm}$ при уменьшении давления аргона от 1 до $0.13 \mathrm{~Pa}$. При этом средний размер зерна $D$ с понижением давления, наоборот, увеличивается с $D \approx 10-20$ до $\approx 35-60 \mathrm{~nm}$, что можно видеть из сравнения электронных изображений на рис. 3.

\section{2. Влияние отжига на микрокристаллическую структуру пленок}

На рис. 1 штриховыми линиями приведены дифрактограммы пленок кобальта толщиной $d \approx 300 \mathrm{~nm}$, осажденных при различных давлениях рабочего газа $P$ 
$a$
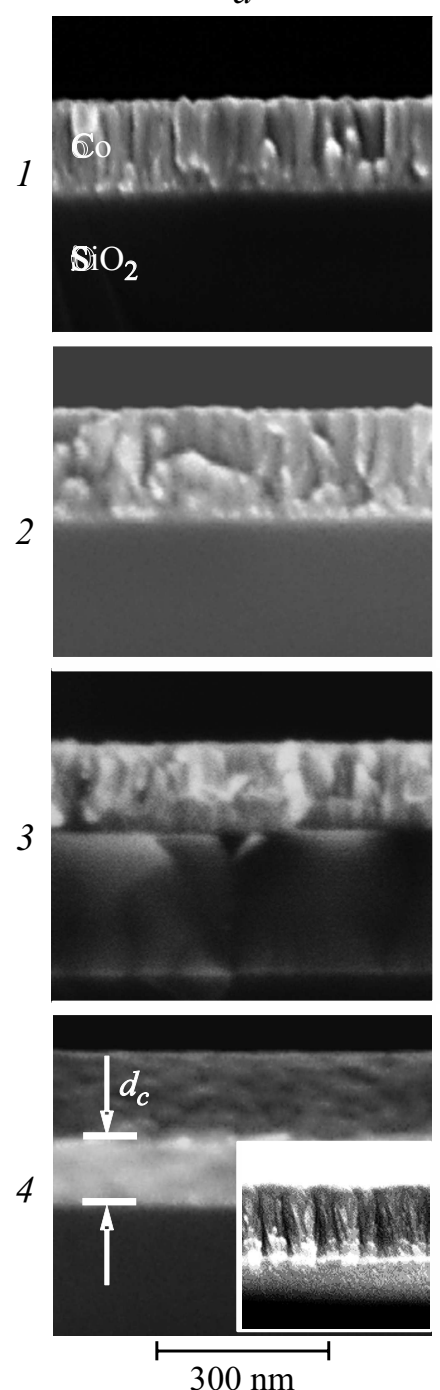

$b$
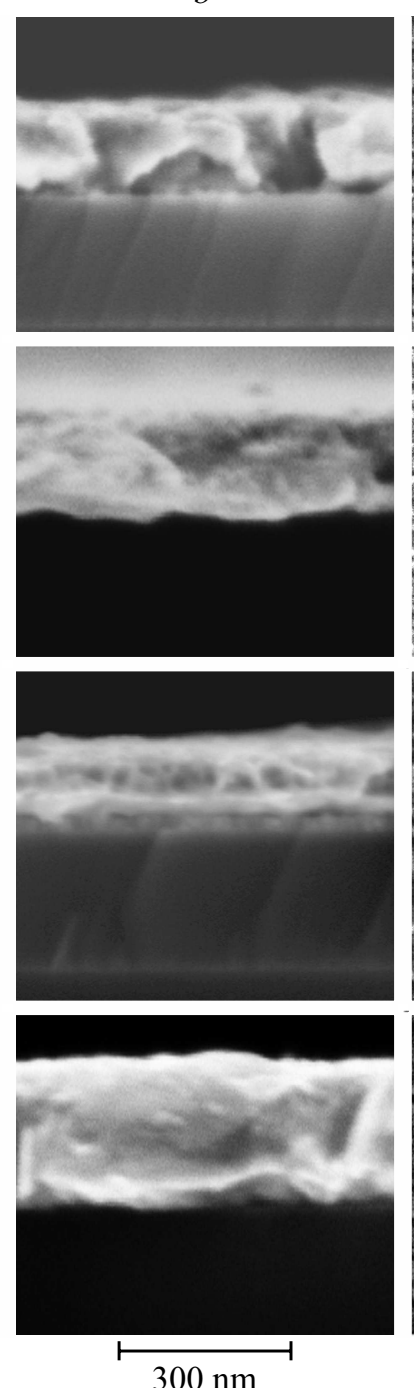

$c$
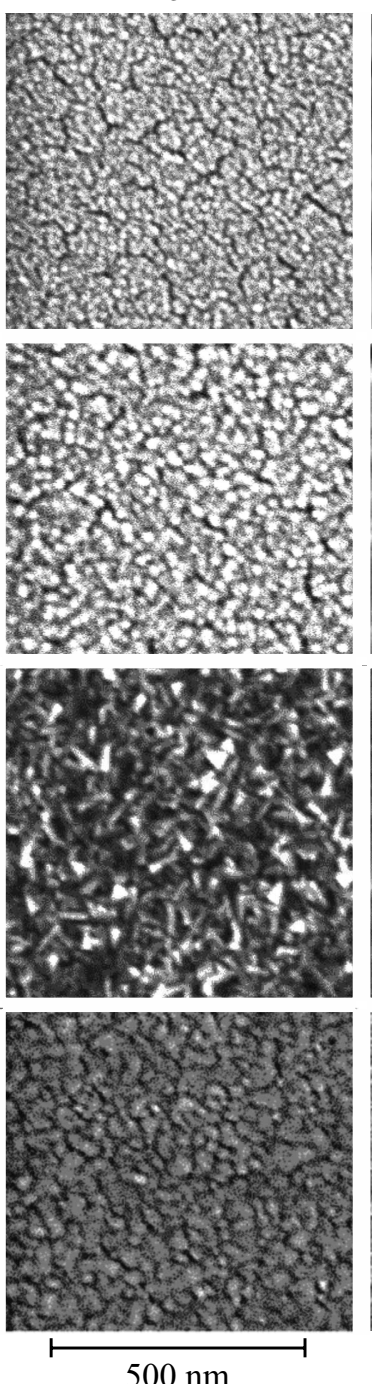
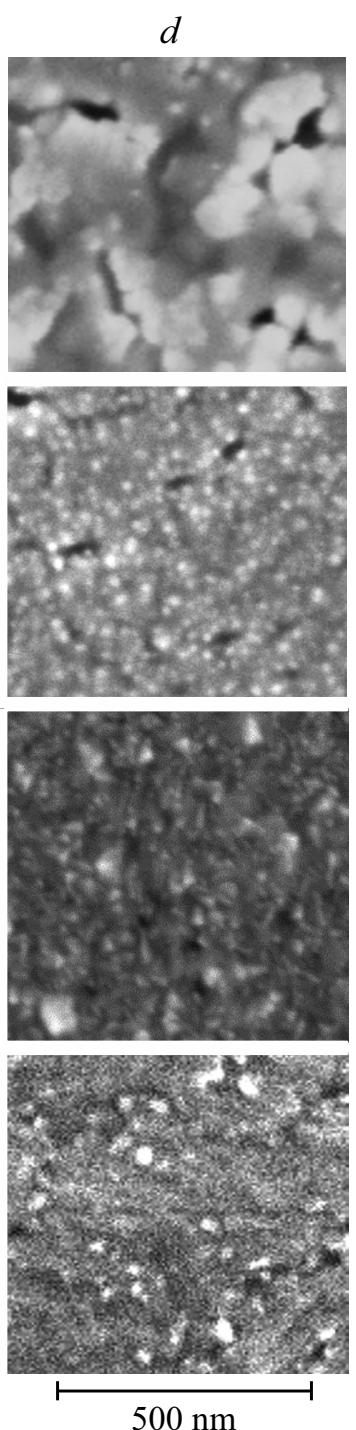

Рис. 3. СЭМ изображения поперечного сечения $(a, b)$ и поверхности $(c, d)$ пленок Со, выращенных при различном давлении аргона $P$, Ра: $1-\approx 1 ; 2-\approx 0.67-0.4 ; 3-\approx 0.18 ; 4-\approx 0.13$ до $(a, c)$ и после отжига при $T_{a} \approx 650 \mathrm{~K}(b, d)$. На вставке к рис. 3, a 4 показано СЭМ изображение поперечного сечения пленки Со, осажденной при $T_{s} \approx 77 \mathrm{~K}, P \approx 0.13$ Ра.

после отжига в вакууме при $T_{a} \approx 650 \mathrm{~K}$ в течении $30 \mathrm{~min}$. Можно видеть, что отжиг пленок, полученных при $P \approx 1-0.26 \mathrm{~Pa}$, значительно улучшает текстуру $\mathrm{Co}(002) / \mathrm{fcc}-\mathrm{Co}(111)$, о чем свидетельствует увеличение в 2.5-4 раза интенсивности дифракционной линии, а также приводит к увеличению однородности пленки за счет увеличения среднего размера зерна до $D \approx 50-100 \mathrm{~nm}$.

Наиболее заметно отжиг влияет на текстуру и микроструктурное строение пленок, полученных при давлении $P \approx 0.18-0.13 \mathrm{~Pa}$ (рис. 1 ). Можно видеть, что интенсивность дифракционного максимума вблизи линии hcp-Co(101) снижается, а фаза fcc-Co(200) становится доминирующей. При этом наблюдаются рефлексы от кристаллических фаз hср-Co(002) и fcc-Co(111). Отметим, что отжиг приводит к заметному смещению дифракционных максимумов относительно положения эталонных образцов, который, например, для fсc-Co(200) фазы проявляется в уменьшении на $0.8 \%$ межплоскостного расстояния в направлении нормали к пленке. В результате отжига микроструктурное строение пленок по толщине становится более однородным - увеличивается средний размер зерна до $D \approx 100-200 \mathrm{~nm}$.

Обсудим механизм преобразования текстуры hcp-Co(101) в fcc-Co(200). Термический нагрев структуры $\mathrm{Co} / \mathrm{Si}$ будет сопровождаться ростом упругих деформаций $\varepsilon$ в пленке Со из-за различия коэффициентов термического расширения Со $\left(\alpha_{\mathrm{Co}} \approx 14.4 \cdot 10^{-6} \mathrm{~K}^{-1} \quad[33]\right)$ и подложки $\mathrm{Si}$ $\left(\alpha_{\mathrm{Si}} \approx 3.5 \cdot 10^{-6} \mathrm{~K}^{-1}[33]\right)$ :

$$
\varepsilon \approx\left(\alpha_{\mathrm{Co}}-\alpha_{\mathrm{Si}}\right) \cdot\left(T_{a}-T_{0}\right) \approx 3.8 \cdot 10^{-3},
$$

где в качестве комнатной температуры $T_{0}$ взято значение $T_{0} \approx 298 \mathrm{~K}$. При этом выигрыш в энергии упругих 
деформаций $\Delta E_{e l}$ между hср-Сo(101) и fcc-Co(200) составит

$$
\begin{aligned}
\Delta E_{e l}= & \left(E_{\mathrm{fcc}(200)}-E_{\mathrm{hcp}(101)}\right) \cdot \varepsilon^{2} \\
& \approx(100-280) \cdot 14.4 \cdot 10^{3} \approx-2.6 \mathrm{MPa},
\end{aligned}
$$

где значения упругих модулей взято из [33]. Если энергия упругих деформаций при смене текстуры пленки hcp-Co(101) на fcc-Co(200) скомпенсирует прирост поверхностной энергии $\Delta \gamma$ пленки, то при температуре отжига $T_{a} \approx 650 \mathrm{~K}$ такая смены текстуры будет возможна. К сожалению, нам не удалось найти в литературных источниках значение поверхностной энергии пленок Со с текстурой hср-Co(101). Поэтому для оценки $\Delta \gamma$ мы воспользуемся значениями поверхностной энергии для пленок hср-Co(002), которые являются наименьшими для кобальта и, следовательно, дадут наибольшее из возможных значений $\Delta \gamma[47]$ :

$$
\Delta \gamma=2 \frac{\gamma_{\mathrm{fcc}}^{(200)}-\gamma_{\mathrm{hcp}}^{(002)}}{d} \approx 1.46 \mathrm{MPa}
$$

где толщина пленки $d$ взята равной $d \approx 300 \mathrm{~nm}$. Из сравнения значений $\Delta \gamma$ и $\Delta E_{e l}$ следует, что при $T_{a} \approx 650 \mathrm{~K}$ деформации пленки способны привести к формированию пленок с текстурой fcc-Co(200). Однако в рамках такого подхода по завершении отжига и возврата температуры пленки к $T_{0} \approx 298 \mathrm{~K}$ текстура пленки должна вернуться к hср-Co(002), что не соответствует результатам на рис. 1. Отметим, что в нашем случае температура отжига $T_{a} \approx 650 \mathrm{~K}$ меньше температуры мартенситного перехода для объемного кобальта $T_{m} \approx 710 \mathrm{~K}$ [57].

Чтобы объяснить стабильность текстуры fcc-Co(200), необходимо учесть пластические деформации кристаллитов, вследствие чего в поведении кристаллической структуры при нагреве и остывании наблюдается гистерезис [58]. В нашем случае упруго-пластический характер деформаций проявляется в несовпадении дифракционных максимумов до и после отжига (рис. 1). Кроме того, это находит отражение в сдвиге дифракционных максимумов относительно эталонных образцов. Учтем далее, что поликристаллические наноматериалы характеризуются многообразием типов ГЗ, в пределах которой расположение атомов теряет периодичность, что ведет к росту энергии границ относительно соседних нанокристаллитов. Поэтому поликристаллическая структура оказывается термодинамически неравновесной, и размер кристаллитов может увеличиваться при отжиге за счет уменьшения общего объема ГЗ. При этом миграция межзеренных границ приводит к росту кристаллитов большего размера за счет поглощения малых зерен, что сопровождается возникновением упруго-пластических деформаций.

Отметим, что возникновение при отжиге упругопластических деформаций отражает изменение эффективного объема пленки при уменьшении числа дефектов упаковки и релаксации энергии ГЗ. Сжатие объема пленок, жестко связанных с подложкой, вызовет в них напряжения

$$
\Delta \sigma=\frac{E_{\mathrm{Co}} \cdot \Delta v}{3(1-v)} .
$$

где $\Delta v=\Delta V / V$ относительное изменение объема, $v \approx 0.32$ - коэффициент Пуссона [57].

Типичные значения концентрации избыточных вакансий $C_{v}$ в распыленных пленках составляет $C_{v} \approx 10^{-4}[59,60]$. Исчезновение избыточных вакансий приведет к относительному уменьшению объема пленки $\Delta V_{v}[42]$ :

$$
\Delta V_{v}=C_{v} \frac{\Omega_{v}}{\Omega}
$$

где $\Omega_{v}$ - объем вакансии, $\Omega$ - атомарный объем. Если энергию образования вакансии принять равной $[61,62]$ $\Delta E_{v} \approx 1.3 \mathrm{eV}$, то исчезновение вакансий приведет к уменьшению плотности энергии пленки на величину $\Delta e_{v}$ :

$$
\Delta e_{v}=\frac{\Delta E_{v} C_{v}}{\Omega} \approx 3 \cdot 10^{6} \frac{\mathrm{J}}{\mathrm{m}^{3}} \approx 3 \mathrm{MPa},
$$

где принято $\Omega_{v}=\Omega \approx 6 \cdot 10^{-30} \mathrm{~m}^{3}$. По порядку величины вызванное исчезновением избыточных вакансий изменение энергии (8) существенно меньше упругих напряжений, вызванных разницей коэффициентов термического расширения при отжиге $(3)\left(\sigma_{\mathrm{hcp}(101)} \approx 1 \mathrm{GPa}\right.$, $\sigma_{\text {fсc(200) }} \approx 380 \mathrm{MPa)} \mathrm{и,} \mathrm{следовательно,} \mathrm{не} \mathrm{может} \mathrm{рассмат-}$ риваться как источник пластических деформаций пленки при отжиге.

Другим источником напряжений, способных привести к пластическим деформациям и вызвать рост зерен, может являться релаксация ГЗ. Если считать, что кристаллиты имеют вид прямоугольных колонн с длиной стороны порядка среднего размера зерна $D \approx 20 \mathrm{~nm}$, то отношение площади ГЗ к объему зерен составит

$$
a_{g b}=\frac{2}{D} \approx 10^{8} \mathrm{~m}^{-1}
$$

Следуя [42], будем считать, что избыточный объем границы на единицу площади $v_{g b, e x c}$ составляет

$$
v_{g b, \mathrm{exc}}=a_{g b} \cdot \Delta V_{\mathrm{exc}} \approx 0.0125 .
$$

где $\Delta V_{\text {exс }}$ - избыточный объем ГЗ на единицу поверхности, по порядку величины совпадающий с атомным радиусом кобальта $\Delta V_{\text {exc }}=0.125 \cdot 10^{-9} \mathrm{~m}$. Это значит, что изменение объема $v_{g b \text {, ехс }}$ составит $v_{g b \text {, ехс }} \approx 0.0125$. Если учесть, что размер зерна $D$ в результате отжига увеличивается в $3-5$ раз $\left(D^{*} \approx(3-5) \cdot D\right.$ (рис. 3$)$, то с учетом (9), (10) получим изменение избыточного объема ГЗ

$$
v_{g b, \mathrm{exc}}=a_{g b} \cdot \Delta V_{\mathrm{exc}} \frac{D^{*}-D}{D^{*}} \approx(0.3-0.8) \cdot 0.0125,
$$

что, согласно (6), приведет к возникновению упругих напряжений $\Delta \sigma \approx(400-500) \mathrm{MPa}$, что сопоставимо 
с величинами напряжений, вызывающих пластические деформации в пленках кобальта при нагреве выше $500 \mathrm{~K}[22]$.

Отметим, что в пленках со смешанной текстурой hcp и fcc нанокристаллиты при отжиге деформируются по-разному, поскольку имеют различные коэффициенты термического расширения $\alpha_{\mathrm{Co}}^{\mathrm{fcc}} \approx 14.4 \cdot 10^{-6} \mathrm{~K}^{-1}$, $\alpha_{\mathrm{Co}}^{\text {hcp }} \approx 13.9 \cdot 10^{-6} \mathrm{~K}^{-1}[33]$. Это различие может приводить к рождению новых дефектов упаковки на ГЗ и влиять на рост кристаллитов.

Рассмотренные выше механизмы деформаций, связанные с разностью коэффициентов термического расширения пленки и подложки, а также исчезновения избыточных вакансий и ГЗ, в условиях жесткой связи с подложкой создают в плоскости пленки деформации разнонаправленного характера. Отжиг приводит к деформациям сжатия, тогда как уменьшение объема - к деформациям растяжения пленки. Дифрактограммы отожженных пленок, выращенных при $P \approx 0.13-0.18 \mathrm{~Pa}$, показывают наличие в плоскости пленки деформаций растяжения для кристаллитов fcc-Co(200). Однако связывать сдвиг положения дифракционной линии hср-Co(200) на 0.8\% относительно эталонного значения только с изменением объема пленки, видимо, не следует. Действительно, отжиг также приводит к падению интенсивности дифракционного максимума hcp-Co(101) и появлению дифракционной линии hсp-Co(002)/fcc-Co(111), что особенно ярко проявляется при $P \approx 0.13 \mathrm{~Pa}$. Поскольку фазы hсp-Co(002)/fcc-Co(111) характеризуются наиболее плотной упаковкой атомов в плоскости пленки, их формирование также может усиливать сдвиг рефлекса от фазы fcc-Co(002).

\section{Заключение}

В настоящей работе исследовано влияние давления рабочего газа $P$ и отжига в вакууме при $T_{a} \approx 650 \mathrm{~K}$ на текстуру и микроструктурное строение пленок кобальта толщиной $d \approx 300 \mathrm{~nm}$, полученных магнетронным распылением на постоянном токе на подложках окисленного кремния. Показано, что при высоком давлении аргона $(0.26 \leq P \leq 1 \mathrm{~Pa})$, когда из-за многократных столкновений с ионами аргона в пространстве между мишенью и подложкой МС адатомов Со оказывается низкой, формируются текстурированные пленки с кристаллической структурой hcp-Co(002)/fcc-Co(111) и столбчатой микроструктурой по толщине. Отжиг таких пленок приводит к увеличению размера зерен и улучшает текстуру hcp-Co(002)/fcc-Co(111). При давлении рабочего газа $0.13 \leq P<0.18 \mathrm{~Pa}$ режим пролета атомов кобальта в пространстве между мишенью и подложкой становится близким к бесстолкновительному, и энергия адатомов Со на поверхности подложки увеличивается. В результате увеличивается как МС адатомов, так и разогрев подложки за счет термализации адатомов. Пленки, полученные при
$0.13 \leq P \leq 0.18 \mathrm{~Pa}$, имеют смешанную кристаллическую фазу: помимо фаз hср-Co(002)/fcc-Co(111) и hсp-Co(101) присутствует кристаллическая фаза fcc-Co(200). Пленки, осаждаемые при $P \approx 0.13 \mathrm{~Pa}$, характеризуются текстурой fcc-Co(200) и неоднородным микроструктурным строением по толщине - на границе с подложкой в слое толщиной $d_{c} \approx 100-130 \mathrm{~nm}$ пленки имеют квазиоднородную микроструктуру, которая на толщинах $d>d_{c}$ переходит в „гранулированную“. Отжиг таких пленок приводит к более однородному микроструктурному строению по толщине пленки, улучшает текстуру $\mathrm{fcc}-\mathrm{Co}(200)$ и приводит к появлению кристаллической фазы fcc-Co(111)/hcp-Co(002).

Полученные результаты могут представлять интерес с точки зрения формирования ориентирующего подслоя $\mathrm{fcc}-\mathrm{Co}(200)$ при создании многослойных структур с перпендикулярной магнитной анизотропией на основе пленок кобальта и благородных металлов [11,12], а также при создании магнето-плазмонных устройств терагерцового диапазона [63].

Работа выполнена при финансовой поддержке РФФИ: грант № 16-37-60052.

\section{Список литературы}

[1] Sbiaa R., Meng H., Piramanayagam S.N. // Phys. Stat. Solid. 2011. Vol. 5. N 12. P. 413-419. DOI: 10.1002/pssr.201105420

[2] Gottwald M., Lee K., Kan J.J., Ocker B., Wrona J., Tibus S., Langer J., Kang S.H., Fullerton E.E. // Appl. Phys. Lett. 2013. Vol. 102. P. 052405-1-4. http://dx.DOI.org/10.1063/1.4791577

[3] Pfau B., Gunter C.M., Guenhrs E., Hauet T., Yang H., Vinh L., Xu X., Yaney D., Rick R., Eisebitt S., Hellwig O. // Appl. Phys. Lett. 2011. Vol. 99. P. 062502-1-3. DOI: 10.1063/1.3623488

[4] Moodera J.S., Kinder L.R., Wong T.M., Mesevey R. // Phys. Rev. Lett. 1995. Vol. 74. P. 3273-3276. DOI: https://DOI.org/10.1103/Phys. Rev. Lett. 74.3273

[5] Tsutsumi K., Haan P., Eisenberg M., Monsma D., Lodder J.C. // JMMM. 1996. Vol. 156. P. 327-328.

[6] Barthelemy A., Mattana R. // Mater. Spintronic. Lect. Notes Phys. 2006. Vol. 697. P. 429-462.

[7] Madani S.S., Zare K., Ghoranneviss M., Elahi A.S. // J. Alloys Compounds. 2015. Vol. 648. P. 1104-1108. http://dx.DOI.org/10.1016/j.jallcom.2015.07.063

[8] Mehedi H.-A., Baudrillart B., Alloyeau D., Mouhoub O., Ricolleau C., Pham V.D., Chacon C., Gicquel A., Lagoute J., Farhat S. // J. Appl. Phys. 2016. Vol. 120. P. 065304-1-11. http://dx.DOI.org/10.1063/1.4960692

[9] Andreev A.S., d'Espinose de Lacaillerie J.-B., Lapina O.B., Gerashenko A. // Phys. Chem. Chem. Phys. 2014. Vol. 17. P. 14598-14604. DOI: 10.1039/C4CP05327C

[10] Ultrathin Magnetic Structure II. / Ed. B. Heinrich, J.A.C. Bland. Berlin: Springer, 1994. Ch. 1. 350 p.

[11] Prinz G.A. // Phys. Rev. Lett. 1985. Vol. 54. P. 1051-1054.

[12] Harp G.R., Farrow R.F.C., Weller D., Rabedeau T.A., Marks R.F. // Phys. Rev. B. 1993. Vol. 48. P. 17538-17544. DOI: https://DOI.org/10.1103/Phys. Rev. B. 48.17538

[13] Methfessel T., Elmers H.J. // Surf. Sci. 2009. Vol. 603. P. 462-468. DOI: 10.1016/j.susc.2008.11.038 
[14] Hao X., Wang Q., Li D., Zhang R., Wang B. // RSC Advances. 2014. Vol. 4. P. 43004-43011. DOI: $10.1039 / \mathrm{c} 4 \mathrm{ra} 04050 \mathrm{c}$

[15] Liu J.-X., Su H.-Y., Sun D.-P., Zhang B.-Y., Li W.-X. // J. Amer. Chem. Soc. 2013. Vol. 135. P. 16284-1-16.

[16] Мягков В.Г., Быкова В.Г., Бондаренко Г.Н., Мягков Ф.В. // ЖТФ. 2002. Т. 72. Вып. 8. С. 122-125.

[17] Yuasa S., Fukushima A., Kubota H., Suzuki Y., Ando K. // Appl. Phys. Lett. 2006. Vol. 89. P. 042505-1-3. DOI: $10.1063 / 1.2236268$

[18] Kim S., Lee S., Kim J., Hong J. // J. Appl. Phys. 2011. Vol. 109. P. 07B766-1-3. DOI: 10.1063/1.3565204

[19] Kurt H., Venkatesan M., Coey J.M.D. // J. Appl. Phys. 2010. Vol. 108. P. 073916-1 -4. DOI: 10.1063/1.3481452

[20] Lau J.W., Mc Michael R.D., Chung S.H., Rantschler J.O., Parekh V., Litvinov D. // Appl. Phys. Lett. 2008. Vol. 92. P. 012506-1-3. DOI: 10.1063/1.2822439

[21] Kumar D., Gupta A. // JMMM. 2007. Vol. 308. P. 318-324. DOI: $10.1016 /$ j.jmmm.2006.06.008

[22] Cabral C., Barmak K., Gupta J., Clevenger L.A., Arcot B., Smith D.A., Harper J.M.E. // J. Vac. Sci. Techn. A. 1993. Vol. 11. P. $1435-1440$. DOI: $10.1116 / 1.578569$

[23] Jergel M., Cheshko I., Halahovets Y., Siffalovic P., Matko I., Senderak R., Protsenko S., Majkova E., Ludy S. // J. Phys. D: Appl. Phys. 2008. Vol. 42. P. $135406-1-8$. DOI: 10.1088/0022-3727/42/13/135406

[24] Morawe Ch., Stierle A., Metoki N., Briihl K., Zabel H. // JMMM. 1991. Vol. 102. P. 223-232.

[25] Shi H., Lederman D. // J. Appl. Phys. 2000. Vol. 87. P. 6095-6097. DOI: $10.1063 / 1.372620$

[26] Джумалиев А.С., Никулин Ю.В., Филимонов Ю.А. // РЭ. 2009. T. 54. C. $347-351$.

[27] Armyanov S. // Electrochimica Acta. 2000. Vol. 45. P. $3323-3335$.

[28] Budendorff J.L., Meny C., Beaurepaire E., Panissod P., Bucher J.P. // Eur. Phys. J. B. 2000. Vol. 17. P. 635-643. https://DOI.org/10.1007/s100510070102

[29] Chioncel M.F., Hyacock P.W. // Chem. Vap. Deposition. 2005. Vol. 11. P. 235-243. DOI: $10.1002 /$ cvde.200406341

[30] Kitakami O., Okamoto S., Shimada Y. // J. Appl. Phys. 1996. Vol. 79. P. 6880-6883.

[31] Ohtake M., Yabuhara O., Higuchi J., Futamoto M. // J. Appl. Phys. 2011. Vol. 109. P. 07C105-1-3. DOI: $10.1063 / 1.3537817$

[32] Kharmouche A., Cherif S.-M., Bourzami A., Layadi A., Schmerber G. // J. Phys. D: Appl. Phys. 2004. Vol. 37. P. 2583-2587. DOI: $10.1088 / 0022-3727 / 37 / 18 / 014$

[33] Lee S.B., Kim D.-I., Kim Y., Yoo S.J., Byun J.Y., Han H.N., Lee D.Y. // Metallurgical and Materials Transactions. 2015. Vol. 46A. P. 1888-1899. DOI: 10.1007/s11661-015-2778-7

[34] Кузьмичев А.И. Магнетронные распылительные системы. Киев: Аверс, 2008. 244 с.

[35] Sakuma H., Tai H., Ishii K. // Trans. Electrical and Electron. Engineer. 2008. Vol. 3. P. 375-378. DOI: 10.1002/tee.20285

[36] Hesemann H.Th., Mullner P., Kraft O., Nowak D., Baker S.P., Finkelstein K., Arzt E. // Scripta Mater. 2003. Vol. 48. P. 1129-1133. DOI: 10.1016/S1359-6462(02)00603-6

[37] Gil W., Görlitz D., Horisberger M., Kötzler J.// Phys. Rev. B. 2005. Vol. 72. P. 134401-1-10. DOI: 10.1103/Phys. Rev. B. 72.134401

[38] Gupta R., Khandelwal A., Avasthi D.K., Nair K.G.M., Gupta A. // J. Appl. Phys. 2010. Vol. 107. P. 033902-1-6. DOI: $10.1063 / 1.3294609$
[39] Itoh K., Ichikawa F., Ishida Y., Okamoto K., Uchiyama T., Iguchi I. // JMMM. 2002. Vol. 248. P. 112-120.

[40] Джуммалиев А.С., Никулин Ю.В., Филимонов Ю.А. // ЖТФ. 2016. Т. 86. Вып. 6. С. 126-131. [Dzhumaliev A.S., Nikulin Yu.V., Filimonov Yu.A. // Techn. Phys. 2016. Vol. 61. N 6. P. $924-928$. DOI: 10.1134/S1063784216060141].

[41] Hoffman R.W. // Thin Sol. Film. 1976. Vol. 34. P. 185-190.

[42] Doerner M.F., Nix W.D. // Critical Rev. Sol.Stat. Mater. Sci. 1988. Vol. 14. P. 225-268. http://dx.DOI.org/10.1080/10408438808243734

[43] Джсумалиев А.С., Никулин Ю.В., Филимонов Ю.А. // ФТТ. 2016. Т. 58. Вып. 6. С. 1206-1215. [Dzhumaliev A.S., Nikulin Yu.V., Filimonov Yu.A. // Phys. Sol. Stat. 2016. Vol. 58. N 6. P. 1247-1256. DOI: $10.1134 / \mathrm{S} 1063783416060135]$.

[44] Alden M., Skriver H.L., Mirbt S., Johansson B. // Surf. Sci. 1994. Vol. 315. P. 157-172.

[45] Alden M., Mirbt S., Skriver H.L., Rosengaard N.M., Johansson B. // Phys. Rev. B. 1992. Vol. 46. N 10. P. 6303-6312.

[46] Yu R., Song H., Zhang X.-F., Yang P. // J. Phys. Chem. B. 2005. Vol. 109. N 15. P. 6940-6943. DOI: 10.1021/jp050973r

[47] Freund L.B., Suresh S. Thin Film materials: Stress, Defect Formation and Surface Evolution. Cambridge: Cambridge University Press, 2003.

[48] Randall M. German. Sintering: From empirical observation to scientific principles. Elsevier/Butterworth-Heinemann, 2014.

[49] Walton D. // J. Chem. Phys. 1962. Vol. 37. P. 2182-2188. DOI: $10.1063 / 1.1732985$

[50] Киттель Ч. Введение в физику твердого тела. М.: Наука, 1978.

[51] Ma Q.-M., Xie Z., Wang J., Liu Y., Li Y.-C. // Phys. Lett. A. 2006. Vol. 358. P.289-296. DOI: $10.1016 /$ j.physleta.2006.05.033

[52] Thompson C.V. // Annu. Rev. Mater. Sci. 2000. Vol. 30. P. 159-190. https://DOI.org/10.1146/annurev.matsci.30.1.159; Thompson C.V., Carel R. // Mater. Sci. Eng. B. 1995. Vol. 32. P. 211-219. https://DOI.org/10.1016/0921-5107(95)03011-5

[53] Pierce D.T., Jimenez J.A., Bentley J., Raabe D., Oskay C., Wittig J.E. // Acta Mater. 2014. Vol. 68. P. 238-253. http://dx.DOI.org/10.1016/j.actamat.2014.01.001

[54] Karunaisiri R.P.U., Bruinsma R., Rudnick J. // Phys. Rev. Lett. 1989. Vol. 62. N 7. P. 788-791.

[55] Thornton J.A. // Ann. Rev. Mater. Sci. 1977. Vol. 7. P. $239-260$.

[56] Langford J.I., Wilson A.J.C. // J. Appl. Cryst. 1978. Vol. 11. P. 102-113. DOI: $10.1107 /$ S0021889878012844

[57] Betteridge W. // Progr. Mater. Sci. 1979. Vol. 24. P. 51-142.

[58] Bidaux J.-E., Schaller R., Benoit W. // J. Phys. Colloques. 1987. Vol. 48. C8-477-482. DOI: https://DOI.org/10.1051/jphyscol:1987874

[59] Lloyd J.R., Nakahara S. // Thin Sol. Film. 1977. Vol. 45. P. 411-420. https://DOI.org/10.1016/0040-6090(77)90282-6

[60] Трусов Л.И., Новиков В.И., Репин И.А., Казилин Е.Е., Ганелин В.Я. // Металлофизика. 1988. Т. 10. С. 104-107.

[61] Matter H., Winter J., Triftshäuser W. // Appl. Phys. 1979. Vol. 20. P. $135-140$.

[62] LaBrosse M.R., Chen L., Johnson J.K. // Modelling and Simulation Mater. Sci. Engineer. 2010. Vol. 18. P. 015008-1-10 . DOI: 10.1088/0965-0393/18/1/015008

63] Gupta B., Pandey S., Nahata A., Sensale-Rodriguez B., Guruswamy S., Nahata A. // Scientific Reports. 2017. Vol. 7. N 12019. P. 1-6. DOI: 10.1038/s41598-017-12369-5 